\title{
Compressive Sampling of Binary Images
}

\author{
Vladimir and Lina Stanković \\ Dept. of Electronic and Electrical Engineering \\ University of Strathclyde \\ Glasgow, U.K. \\ \{vladimir,lina\}.stankovic@eee.strath.ac.uk
}

\author{
Samuel Cheng \\ School of ECE \\ University of Oklahoma \\ Tulsa, OK, US \\ samuel.cheng@ou.edu
}

\begin{abstract}
Compressive sampling is a novel framework that exploits sparsity of a signal in a transform domain to perform sampling below the Nyquist rate. In this paper, we apply compressive sampling to reduce the sampling rate of binary images. A system is proposed whereby the image is split into non-overlapping blocks of equal size and compressive sampling is performed on selected blocks only using the orthogonal matching pursuit technique. The remaining blocks are sampled fully. This way, the complexity and the required sampling time is reduced since the orthogonal matching pursuit operates on a smaller number of samples, and at the same time local sparsity within an image is exploited. Our simulation results show more than $20 \%$ saving in acquisition for several binary images.
\end{abstract}

\section{Introduction}

Compressive sampling is gaining much attention recently. Simply put, it is a statistical technique for data acquisition and estimation that aims to sample signals sparsely in transform domains. The sparse samples may be used to estimate the original signal under certain conditions. This makes compressive sampling very attractive for digital image and video applications, where the Nyquist rate is so high that compressing the sheer volume of samples is a problem for transmission or storage. The process of compressive sampling replaces conventional sampling and reconstruction with a more general linear measurement scheme with an optimization procedure to acquire a subset of signals within a source at a rate that is significantly below Nyquist.

A number of theoretical contributions have appeared on compressive sampling (see $[1,2,3]$ ) over the past few years. Yet, only few papers address day-to-day practical situations, for example, analog-to-information converter [4] and onepixel camera [5]. In this paper, we consider simple applica- tions which would benefit tremendously from compressive sampling in order to simplify the sampling process and possibly the compression process.

Typical applications would range from acquisition of image/video from an array of cameras monitoring a large area. Solutions would range from extraction of relevant data to excellent compression schemes. Alternatively, we propose simply acquiring enough samples (well below Nyquist) to enable reconstruction with good perceptual quality. The challenge is to predict which scenes are sparse in the transform domain. Generally, binary images or video tend to possess many (generally greater than $50 \%$ ) zero coefficients (in transform or pixel domain) in static backgrounds or very slow motion. An example would be monitoring the flight pattern of birds in a section of sky - besides the centre block of the image or video, everything else would be sparse. Similarly another application scenario gaining much attention recently is automated pedestrian sensing and tracking for security, urban planning, and the retail industry. A common approach is to use CCTV and visible image processing, but this involves complicated scene interpretation. Infrared cameras are relatively low-cost and with lower resolution but it is possible to perform tracking of pedestrians, and collect biometric data, such as gait measurement [6]. However, sampling and reconstruction of the footage is an expensive task. Thus, we exploit the fact that the scene around the pedestrian will be mostly static and hence lend itself to compressive sampling.

However, this is not a simple task because only fractions of the source will be sparse. We introduce a simple method whereby the source, say an image, is partitioned into equally sized blocks. Sparse blocks would be sampled compressively, and the remaining blocks which contain the important information sampled fully. The size of the blocks will depend on the location of 'sparse' areas within the source. We show that is possible to significantly reduce the total number of samples using compressive sampling. Reconstruction of the sparsely sampled blocks is achieved from the orthogonal matching pursuit algorithm 
[7], which is suboptimal but practical due to its relatively lower complexity than other proposed reconstruction methods $[2,1,8,9]$.

This paper is proposed as follows: Section II provides an overview of compressive sampling, Section III describes our proposed system from acquisition to reconstruction, results using binary images are presented in Section IV, and we conclude in Section V.

\section{Compressive Sampling}

Compressive sampling or compressed sensing $[1,2]$ is a novel framework that enables sampling below Nyquist rate, without (or with minimum) sacrificing reconstruction quality by exploiting sparsity of the signal in some domain. In this section we briefly review compressed sampling following closely notation of [3].

Let $\mathbf{x}=\{x[1], \ldots, x[N]\}$ be a set of $N$ samples of a real-valued, time-discrete random process $X$. Let $\mathbf{s}$ be representation of $\mathrm{x}$ in the $\boldsymbol{\Psi}$ domain, that is:

$$
\mathbf{x}=\boldsymbol{\Psi} \mathbf{s}=\sum_{i=1}^{N} s_{i} \psi_{i}
$$

where $\mathbf{s}=\left\{s_{1}, \ldots, s_{N}\right\}$ is an $N$-vector of weighted coefficients $s_{i}=<\mathbf{x}, \psi_{i}>$, and $\boldsymbol{\Psi}=\left[\psi_{1}\left|\psi_{2}\right| \cdots \mid \psi_{N}\right]$ is an $N \times N$ basic matrix with $\psi_{i}$ being the $i$-th basic column vector.

We say that vector $\mathrm{x}$ is $K$-sparse in the domain $\Psi$, $K<<N$, if only $K$ out of $N$ coefficients of $\mathbf{s}$ are nonzero. Sparsity of a signal is used for compression in conventional transform coding, where the whole signal is first acquired (all $N$ samples of $\mathbf{x}$ ), then the $N$ transform coefficients $\mathbf{s}$ are obtained via $\mathbf{s}=\Psi^{T} \mathbf{x}$, and then $N-K$ (or more in the case of lossy compression) coefficients of $\mathbf{s}$ are discarded and the remaining are encoded. The most appealing example is Discrete Cosine Transform (DCT) in image coding.

Note that huge redundancy is present in the acquisition since large amount of data are discarded (and hence not used at all), because they carry negligible energy. The main idea of compressive sampling is to remove this "sampling redundancy" by requiring only $M$ samples of the signal, where $K<M<<N$. Let $\mathbf{y}$ be a $M$-length measurement vector given by:

$$
\mathbf{y}=\mathbf{\Phi} \mathbf{x}
$$

where $\boldsymbol{\Phi}$ is an $M \times N$ measurement matrix. The above expression can be written in terms of $\mathbf{s}$ as:

$$
\mathrm{y}=\boldsymbol{\Phi} \Psi_{\mathrm{s}}
$$

It has been shown in $[2,1]$ that signal $\mathrm{x}$ can be recovered losslessly from $M \approx K$ or slightly more measurements (vector $\mathbf{y}$ in (3)) if the measurement matrix $\boldsymbol{\Phi}$ is properly designed, so that $\boldsymbol{\Phi} \boldsymbol{\Psi}$ satisfies the so-called restricted isometry property [2]. This will always be true if $\boldsymbol{\Phi}$ and $\boldsymbol{\Psi}$ are incoherent, that is the vectors of $\boldsymbol{\Phi}$ cannot sparsely represented basic vectors and vice versa.

It was further shown in [1, 2, 3] that a Gaussian matrix $\boldsymbol{\Phi}$ satisfies the above property for any (orthonormal) $\boldsymbol{\Psi}$ with high probability if $M \geq c K \log (N / K)$ for some small constant $c$. Thus, one can recover $N$ measurements of $\mathbf{x}$ with high probability from only $M \approx c K \log (N / K)<<N$ random Gaussian measurements $\mathbf{y}$ under the assumption that $\mathbf{x}$ is $K$-sparse in some domain $\Psi$. Note that it is not known in advance which $s_{i}$ coefficients are zeros, or which $x[i]$ samples are not needed.

Unfortunately, reconstruction of $\mathbf{x}=\{x[1], \ldots, x[N]\}$ (or equivalently, $\mathbf{s}=\left\{s_{1}, \ldots, s_{N}\right\}$ ) from vector $\mathbf{y}$ of $M$ samples is not trivial. The exact solution [1, 2, 3] is NP-hard and consists of finding the minimum $l_{0}$ norm (the number of non-zero elements). However, excellent approximation can be obtained via the $l_{1}$ norm minimization given by:

$$
\hat{\mathbf{s}}=\arg \min \left\|s^{\prime}\right\|_{1}, \quad \text { such that } \boldsymbol{\Phi} \Psi \mathbf{s}^{\prime}=\mathbf{y} .
$$

This convex optimization problem, namely, basis pursuit $[1,2]$, can be solved using a linear program algorithm of $O\left(N^{3}\right)$ complexity. In contrast to $l_{0}$ norm minimization, the $l_{1}$ norm minimization usually requires more than $K+1$ measurements. Due to complexity and low speed of linear programming algorithms, faster solutions were proposed at the expense of slightly more measurements, such as matching pursuit, tree matching pursuit [8], orthogonal matching pursuit [7] (used in this paper), and group testing [9].

\section{Compressive Sampling of Binary Images}

In this section, we describe our system, shown in Figure 1 , for compressive sampling of binary images. The system performs compressive sampling on the image blocks using orthogonal matching pursuit (OMP) [7]. We use i.i.d. Gaussian measurement matrix $\boldsymbol{\Phi}$ and unit transform $\boldsymbol{\Psi}$ (hence, we exploit sparsity of the image in the pixel domain).

The OMP algorithm is an efficient solution for signal recovery that is easy to implement. It is of $O(M N K)$ complexity, and requires $M \approx 2 m \log N$ measurements in $99 \%$ of time. The algorithm has $K$ iterations, and in each iteration it calculates $N$ inner products between $M$-length vectors and finds the maximum. Thus, when $M$ is large the algorithm is slow and impractical for online applications.

To reduce the complexity we split the image into $B$ nonoverlapping blocks of equal size. Then, $B_{1} \leq B$ blocks are picked for compressive sampling. The remaining blocks are sampled in the conventional way (that is, all pixels are collected). 


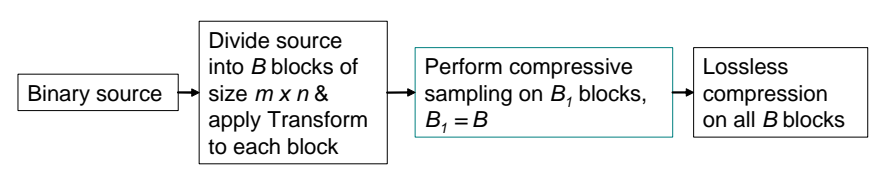

Reconstructed

Source

\section{Inverse transform}

on $B$ blocks

\section{Figure 1. Block diagram of the proposed sys- tem.}

The OMP algorithm is now used for each of $B_{1}$ selected blocks independently after transform. Using this method, besides reducing the required time, we also exploit local sparsity within image in a more efficient manner. That is, the parts of the image that are non-sparse are not sampled in the compressed way, and the OMP algorithm is used only for the regions that are estimated to be sparse. For example, the background is a good candidate for compressive sampling whereas areas with many details are not. After (compressive) sampling conventional lossless compression can be applied.

The main problem that arises is the selection of the blocks to be compressively sampled. In wireless sensor applications (and in video acquisition), where a sensor acquires images in real time, the selection can be based on previous images.

Note that any transform can be applied under the condition that the signal is sparse in the transform domain.

\section{Results}

In this section we report our experimental results. We use the system described in the previous section with three binary images of different sizes.

In the first experiment we use a small size image $(16 \times 8$ pixels) shown in Figure 2 (top left). The image is not split into blocks, that is, compressive sampling was applied to the whole image. The image stream contains $N-K=107$ zeros (and $K=21$ ones), hence it is sparse in the pixel domain. We sampled the image in the pixel domain using the Gaussian measurement matrix. The results obtained for three different measurements $M$ are shown in Figure 2. It can be seen from the figure that $M=100<N=128$ measurements are sufficient to perfectly recover the image, which yields to roughly $21 \%$ savings in acquisition. When 80 and 90 measurements were used for reconstruction, the orthogonal matching pursuit was not able to correctly recover the whole image. As expected with the OMP, $M \approx 2 K \log N \approx 93$

(see Section III), the number of measurements needed is much higher than the theoretical value of $K+1=22$ with $l_{0}$ norm minimization.
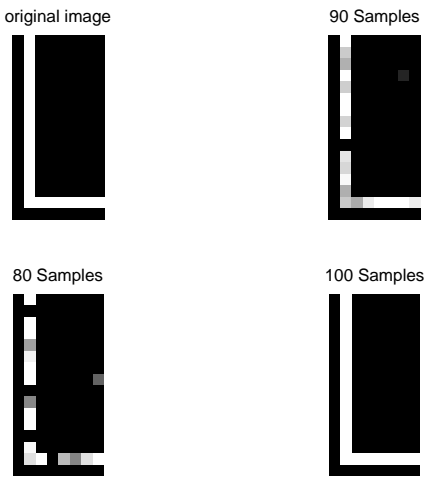

Figure 2. Results with the "L" image of 16 $\times 8$ pixels: original image (top left) and reconstructions with 80 measurements (bottom left), 90 measurements (top right), and 100 measurements (bottom right).

In the next experiment, we use the "Lancaster Logo" image of $128 \times 64$ pixels (hence, the total number of bits is $N=8,192$ ). The total number of zeros is 6,311 . We split the image into $B=32$ blocks each of size $16 \times 16$ pixels and show in Figure 3 the distribution of the number of zeros over all $B$ blocks. It can be seen that the image contains many blocks that are not sparse enough (with number of zeros beyond $20 \%$ ), for which compressive sampling is not effective. Thus, we perform compressive sampling on $B_{1}<B$ blocks only. Remaining $B-B_{1}$ blocks are sampled fully (using $256=16 \times 16$ measurements with unity measurement matrix). The next figure shows our obtained results as residual bit error rate (BER) after reconstruction as a function of $B-B_{1}$, that is, the number of blocks on which compressive sampling was not applied. The number of measurements $M$ (for each of $B_{1}$ compressive sampled block) is 200,220 , and $240<N=16 \times 16=256$. The first conclusion from the figure is that error-free recovery is possible with $B_{1}=10$ compressive sampled blocks. The second conclusion is that $M=200$ measurements are sufficient.

In the last experiment we use the "Neighborhood_watch_bw" image of size $140 \times 200$ pixels shown in Figure 6 (top left). We split the image into $B=280$ $10 \times 10$ blocks. Again, we used compressive sampling for $B_{1}<B$ blocks. The results are presented in Figure 5 as BER vs. $B-B_{1}$ for the number of measurements $M$ equal to 60,80 , and 90 . It can be seen from the figure that 


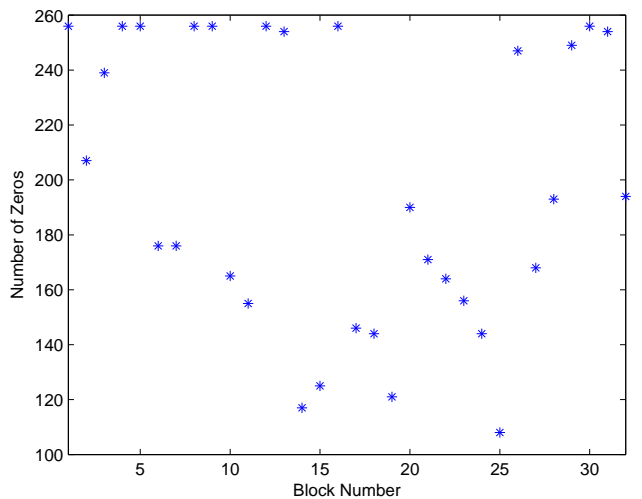

Figure 3. Distributions of the number of zeros over $B=32$ blocks for the "Lancaster image" of size $128 \times 64$ pixels.

roughly 30 blocks were efficiently sampled compressively for the number of measurements $M=60$.

Figure 6 shows the three reconstructed images for the number of measurements $M$ equal to 20,50, and 80. Only blocks with at least $70 \%$ of zeros were compressive sampled ( $B_{1}=118$ in total). The improvement in the reconstruction quality as the number measurements $M$ increases can be observed.

\section{Conclusions and Future Work}

We have applied the novel concept of compressive sampling on a practical problem of sampling binary images. Due to the complexity of compressive sampling algorithms and varying sparsity within an image, we split the image into non-overlapping blocks, and only perform compressive sampling on some of the blocks, while the remaining blocks were sampled fully. Experimental results show great potential for compressive sampling for binary image acquisition. In future work, we will extend our system for gray-scale images and compressive sampling in other domains.

\section{References}

[1] D. Donoho, "Compressed sensing," IEEE Trans. Inform. Theory, vol. 52, pp. 1289-1306, April 2006.

[2] E. Candès, J. Romberg, and T. Tao, "Robust uncertainty principles: Exact signal reconstruction from highly incomplete frequency information," IEEE Trans. Inform. Theory, vol. 52, pp. 489-509, Feb. 2006.

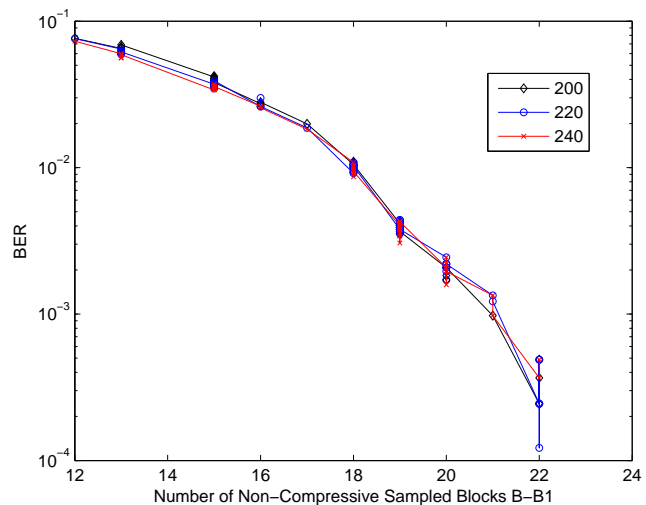

Figure 4. BER vs. the number of blocks for which compressive sampling was not applied for the "Lancaster Logo" image. Three different number of measurements are compared.

[3] R.G. Baraniuk, "Compressive Sensing [Lecture Notes]," IEEE Signal Processing Magazine, vol. 24, pp. 118-121, July 2007.

[4] S. Kirolos, J. Laska, M. Wakin, M. Duarte, D. Baron, T. Ragheb, Y. Massoud, and R.G. Baraniuk, "Analogto-information conversion via random demodulation," in IEEE Dallas Circuits and Systems Workshop, Oct. 2006.

[5] M. Wakin, J. Laska, M. Duarte, D. Baron, S. Sarvotham, D. Takhar, K. Kelly, and R.G. Baraniuk, "Compressive imaging for video representation and coding," in Proc. Picture Coding Symposium (PCS), Beijing, China, April 2006.

[6] T. Chamberlain, A.Armitage, M Rutter, and D. Binnie, "Pedestrian sensing with feature extraction", ITS World Congress London, 2006.

[7] J. Tropp and A.C. Gilbert, "Signal recovery from partial information via orthogonal matching pursuit," preprint 2005.

[8] M.F. Duarte, M.B. Wakin, and R.G. Baraniuk, "Fast reconstruction of piecewise smooth signals from random projections," in Proc. SPARSO5 Signal Processing with Adaptive Sparse Structured Representations, Rennes, France, Nov. 2005.

[9] G. Cormode, S. Muthukrishnan, "Towards an algorithmic theory of compressed sensing," DIMACS Tech. Report 2005-40, 2005. 


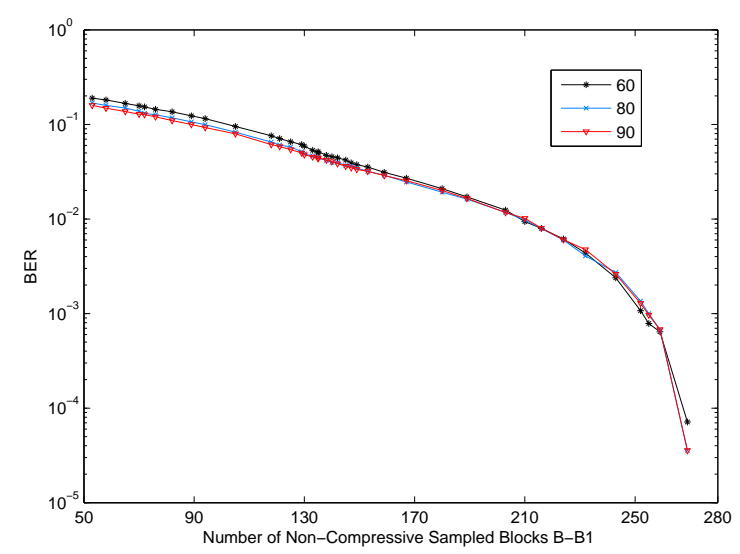

Figure 5. BER vs. the number of blocks for which compressive sampling was not applied for the "Neighborhood_watch_bw" image. Three different number of measurements are compared.
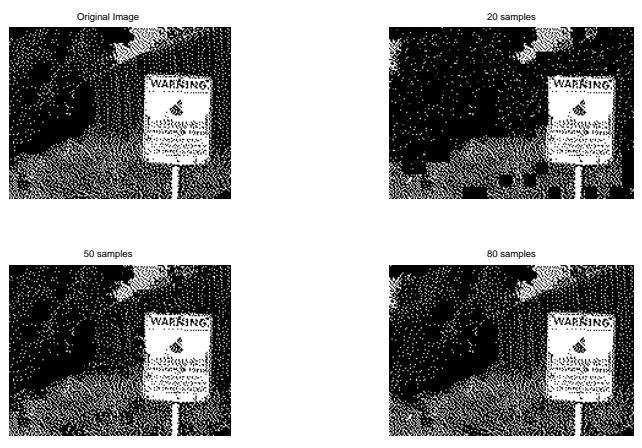

Figure 6. Results with the "Neighborhood_watch_bw" image of $140 \times 200$ pixels: original image (top left) and reconstructions with 20 measurements (bottom left), 50 measurements (top right), and 80 measurements (bottom right). 